\title{
Reflexiones literarias de escritores en lengua alemana desde el exilio en México en la revista Freies Deutschland (Alemania Libre) ${ }^{1}$
}

\author{
Teresa CAÑADAS GARCÍA \\ Departamento de Filología Alemana \\ Universidad Complutense de Madrid \\ tcanadas@ucm.es
}

\begin{abstract}
RESUMEN
Los escritores alemanes que llegaron durante el exilio a México lograron fundar una editorial, un club teatral, asociaciones culturales y varios periódicos y revistas. Una de ellas fue la revista Freies Deutschland (Alemania Libre), de contenido político pero también artístico y literario. En ella se publicaron, de mano de los propios escritores alemanes, numerosas reflexiones en torno a la producción literaria en el exilio que nos ayudan a entender en qué medida la guerra influyó en los escritores de habla alemana y, a su vez, en los procesos de creación literaria de esa época.
\end{abstract}

Palabras clave: exilio, concepción literaria, Alemania, México, prensa del exilio.

\section{Literary reflections of German-speaking writers from exile in Mexico in the journal Freies Deutschland (Free Germany)}

\begin{abstract}
German writers who arrived in exile to Mexico succeeded in founding a publishing house, a theater club, cultural associations and some newspapers and journals. One of them was the journal Freies Deutschland (Free Germany) that contained mainly political but also artistic and literary articles. A lot of reflections on literary production in exile were published here by German-speaking writers; that helps us to understand to what extent war influenced them and therefore the process of literary creation of that time.
\end{abstract}

Keywords: exile, literary conception, Germany, Mexico, press in exile.

${ }^{1}$ Las citas que hay a lo largo del artículo procedentes de la revista Freies Deutschland han sido traducidas por la autora. 
Desde la llegada de Hitler al poder en 1933, comenzaron a tener lugar en Alemania una serie de acontecimientos, cada vez más graves, que desembocarían en la Segunda Guerra Mundial.

En la noche del 27 de febrero de 1933 ardió el Parlamento Alemán; dado que las autoridades declararon culpables a los grupos de izquierdas, sobre todo al KPD (Partido Comunista de Alemania), se comenzó a arrestar a intelectuales y políticos de ese partido y entró en vigor una ley de emergencia, el «Decreto del Presidente del Reich para la Protección del Pueblo y del Estado» (Verordnung des Reichspräsidenten zum Schutz von Volk und Staat) que prohibía el derecho de secreto postal, la libertad de opinión, la libertad de prensa, la libertad de asociación, etc. y que obligaba, por tanto, a la disolución de todos los partidos que no fueran el Partido Nacionalsocialista Obrero Alemán.

Ante estas circunstancias, los opositores al régimen y todas las personas perseguidas buscaron la manera de escapar de las garras nazis huyendo del país a nuevos lugares donde no estuvieran amenazados. Como primera opción primó la elección de países cercanos a Alemania, con la esperanza de que el terror acabara pronto y pudieran regresar. Pero no todos pudieron huir o prefirieron, quizás, quedarse clandestinamente en Alemania (Stephan 1979).

El estallido de la Segunda Guerra Mundial y el consecutivo avance de las tropas nazis por Francia, Dinamarca, Noruega, Países Bajos, Grecia, Yugoslavia y la Unión Soviética obligó a que aquellos que se habían exiliado en esos países ahora invadidos, tuvieran que buscar nuevos lugares de refugio. El horizonte de un lugar donde establecerse con mayor seguridad se alejó hasta otros continentes, como en el caso de América.

De los alemanes que huyeron, principalmente fueron los judíos o los intelectuales de renombre internacional los que consiguieron llegar a Estados Unidos, pero también muchos escaparon a países de habla hispana y, en concreto al país del que hablamos en este trabajo: México.

Desde la primavera de 1939, el presidente mexicano, Lázaro Cárdenas, hizo una oferta de asilo a los republicanos españoles que habían huido a Francia y también a los luchadores extranjeros en la Guerra Civil que hubieran adquirido la nacionalidad española durante la contienda. Sin embargo, ante la grave situación y ante la insistencia de algunos fugitivos políticos no españoles que también se encontraban en peligro, los diplomáticos mexicanos en Europa intentaron hacer lo posible por salvarlos. Más adelante, cuando Francia capituló, el gobierno de Lázaro Cárdenas cerró un acuerdo con la Francia de Vichy en agosto de 1940 para intentar salvar a tantos fugitivos como le fuera posible. Las estadísticas de inmigración mexicanas recogen que entre 1935 y 1942 llegaron al país 6.168 inmigrantes no españoles (Pohle 1986: 5).

Salvadas las numerosas dificultades para llegar a México, el grupo de exiliados de habla alemana trató en primer lugar de buscar la manera de salir adelante con diversos trabajos y, ayudados por algunas asociaciones, ver cómo combatir el fascismo desde su nuevo destino, defender la Alemania humanista que estaba siendo arrollada y ultrajada por el nazismo y desenmascarar la cruel Alemania hitleriana.

El ámbito cultural mexicano, donde estaban asentados ahora los exiliados alemanes, fue el marco en el que tuvieron que moverse para conseguir sus objetivos de 
lucha contra el fascismo, de propagación del humanismo alemán y de mantenimiento de una postura de unidad cultural fuerte como símbolo eficaz de defensa de la Alemania que representaban. Por ello, organizaron en México actividades de naturaleza política y cultural con los medios que encontraron a su alcance.

El grupo más productivo y unido de los exiliados de habla alemana en México fue el Movimiento Alemania Libre (Bewegung Freies Deutschland) ${ }^{2}$, que se fundó en enero de 1942. Previamente, cuando en octubre de 1941 llegó un considerable número de comunistas a México en el barco Serpa Pinto, entre los cuales había escritores, periodistas y publicistas, los exiliados vieron la oportunidad idónea para hacer efectiva la lucha contra el fascismo. De este modo, los planes de creación de una revista en lengua alemana llamada Freies Deutschland (FD) se hicieron realidad y se logró publicar en México D. F. el primer número en noviembre de 1941.

Debido a las leyes mexicanas, el regente de la revista fue el escritor, diplomático, profesor y rector de la Universidad Nacional de México Antonio Castro Leal, quien en el primer número explicó: «La revista Alemania Libre es el órgano de algunos espíritus generosos que desean ver a su patria, Alemania, salvada para siempre de la dictadura nazi» (Castro Leal 1941: 2).

Se publicó mensualmente desde noviembre de 1941 hasta junio de 1946 y se distribuyó su venta oficialmente en Estados Unidos, Argentina, Chile, Venezuela, Inglaterra, Brasil, Colombia, Costa Rica, Ecuador y Uruguay.

A partir del 17 de enero de 1942 se publicó también el boletín Alemania Libre. Boletín semanal de información antinazi, en español, para luchar contra la quinta columna y para informar a los lectores de habla española sobre la oposición y resistencia contra el fascismo alemán.

En el primer número de $F D$, el escritor Ludwig Renn llamaba a todos los grupos antifascistas americanos a crear un frente de libertad nacional, un paso más allá de lo que hasta ahora había sido la lucha fallida por crear un frente popular en París y que será uno de los objetivos de $F D$.

La revista tenía un marcado carácter político y literario y contó con la colaboración de los propios exiliados de habla alemana en México (Paul Merker, Ludwig Renn, Alexander Abusch, Paul Mayer, Anna Seghers, André Simone, Theodor Balk, Leo Katz, Paul Westheim), pero también con la colaboración de exiliados en Estados Unidos (Thomas Mann, Heinrich Mann, Lion Feuchtwanger, Oskar Maria Graf, F. C. Weiskopf, Albert Norden, Alfred Kantorowicz), y la de escritores exiliados en la Unión Soviética (Friedrich Wolf, Willi Bredel, Erich Weinert) o importantes escritores o políticos mexicanos, latinoamericanos y españoles (Vicente Lombardo Toledano, José Bergamín, Pablo Neruda). Es, por tanto, una revista representativa para

\footnotetext{
${ }^{2}$ Se definía como organización alemana de todos los opositores a Hitler sin distinción de procedencia religiosa o política y se presentó a la luz pública el 1 de febrero de 1942. El programa de acción se resumía en la persecución del fascismo alemán a través de la defensa y el fomento de la cultura alemana humanista, colaborando a su vez con todos los movimientos nacionales mexicanos que lucharan contra el fascismo y siendo leales al país de acogida, México.
} 
conocer no solo el punto de vista de los alemanes exiliados en México, sino también de los alemanes exiliados en otros países.

Los contenidos de la revista estaban coordinados por distintos encargados, según su especialidad, procedencia o profesión: Bodo Uhse se ocupaba de la parte cultural, Ludwig Renn de las noticias y evolución de la contienda, Leo Katz y Theodor Balk comentaban los acontecimientos en el sureste de Europa, Bruno Frei se encargaba de los aspectos relativos a Austria y André Simone y Alexander Abusch se ocupaban de la política mundial escribiendo artículos bajo las directrices que indicaba el político Paul Merker y que, a su vez, recibía en ocasiones desde la Unión Soviética.

La revista $F D$ tuvo unas secciones fijas como $F D$ berichtet ( $F D$ informa), $F D$ liest ( $F D$ lee) y $F D$ hört ( $F D$ escucha) en las que se contaban noticias de hechos que sucedían en México o sobre exiliados de otros lugares del mundo. Secciones que aparecieron de manera intermitente fueron: Das Buch in unserer Zeit (El libro en nuestro tiempo), con reseñas sobre los libros que se publicaban en México, reflexiones literarias y recomendaciones de lecturas; Bewegung Freies Deutschland (Movimiento Alemania Libre), que contenía noticias sobre dicha agrupación y la relación que mantenían con miembros antifascistas de otros movimientos o países; Stimmen der Vergangenheit (Voces del pasado), en la que se honraba y rememoraba a intelectuales alemanes del pasado y Briefe an die Redaktion (Cartas a la redacción), que incluía cartas de los lectores de la revista.

Desde enero de 1946 y hasta el final de su existencia, se cambió el nombre de la revista por Neues Deutschland (Nueva Alemania), haciendo referencia a la Alemania que surgió tras la guerra.

Quiero estudiar ahora las reflexiones en torno a la literatura que aparecieron en la revista Freies Deutschland, en concreto las publicadas en la sección titulada Das Buch in unserer Zeit (El libro en nuestro tiempo). Esta sección se editó en la revista mensualmente, desde marzo de 1943 hasta mayo de 1946.

Además de incluir recensiones y reseñas de libros que se publicaban tanto en México como en otros países (Estados Unidos o la Unión Soviética), se incluyeron algunos artículos de sumo interés para comprender cómo se entendía en tales circunstancias, desde el exilio causado por la guerra, la labor del escritor y la situación de la literatura, en concreto, de la literatura de habla alemana. Los artículos seleccionados de la citada sección que tendré en cuenta son:

1. «El escritor y la guerra» («Der Schriftsteller und der Krieg»), Bodo Uhse, febrero de 1943.

2. «El horror mira en derredor. Comentarios sobre la literatura alemana de guerra». («Das Grauen sieht sich um. Bemerkungen zur deutschen Kriegsliteratur»), F. C. Weiskopf, agosto de 1943.

3. «Responsabilidad y obligación» («Verantwortung und Pflicht»), Bodo Uhse, octubre de 1943.

4. «Problemas del trabajo del escritor en el exilio» («Arbeitsprobleme des Schriftstellers im Exil», Lion Feuchtwanger, marzo de 1944. 
5. «Obligaciones del escritor» («Pflichten des Schriftstellers»), Ilja Ehrenburg, mayo de 1944.

6. «Sobre el presente y futuro de la literatura alemana» («Von Gegenwart und Zukunft der deutschen Literatur»), Bodo Uhse, julio de 1944.

7. «Una reflexión sobre el arte en la guerra» («Eine Betrachtung über Kunst im Krieg»), J. R. Becher, septiembre de 1944.

8. «Libro y editorial en la Alemania que viene» («Buch und Verlag im kommenden Deutschland»), Walter Janka, marzo de 1945.

9. «Literatura alemana de postguerra» («Deutsche Nachkriegsliteratur»), Max Zimmering, septiembre de 1945.

10. «Logros de la editorial El Libro Libre» («Leistungen des Verlages El Libro Libre»), Paul Mayer, mayo de 1946.

En ellos el arte, la literatura, el artista y la guerra son los temas principales; ciertas ideas y aspectos se repiten constantemente, lo que ayuda a ver cómo percibían los propios artistas y escritores su esencia y su ser en esos años de guerra y de exilio y a entrever «reflejos de guerra» en los artículos publicados en la revista $F D$ y, por tanto, en la concepción literaria de aquel tiempo.

La perspectiva es la de escritores de habla alemana que publicaban en una revista de ideología comunista en el exilio en México. Algunos de los rasgos que van a destacar en los citados artículos pueden encontrarse en el modus operandi del comunismo internacional en torno a los años de la guerra en lo referente al arte, a la intelectualidad y, más concretamente, a la literatura.

Estas líneas de actuación y de lucha impulsadas por el comunismo nacían, se desarrollaban y se difundían por un lado en los congresos mundiales del Comintern, pero también en congresos más pequeños y específicos como el celebrado en 1934, I Congreso de Escritores Soviéticos, promovido por la Unión de Escritores Soviéticos y en el que participaron un grupo de alemanes, entre ellos Oskar Maria Graf, Gustav Regler, Johannes R. Becher o Wieland Herzfelde.

Otros encuentros fueron también importantes, como la Conferencia de Bruselas promovida por el Partido Comunista Alemán y que tuvo lugar del 3 al 15 de octubre de 1935 en Moscú; en ella se adaptaron las resoluciones del VII Congreso Mundial del Comintern de agosto de 1935 a la situación de Alemania, se llamaba a la defensa de la cultura, ciencia, lengua y artes alemanas frente al fascismo, al imperialismo y al militarismo y a dar nuevos impulsos humanistas que facilitaran al pueblo trabajador alemán el camino hacia el socialismo. En esta línea se trabajó también en la Conferencia de Berna organizada por el KPD del 30 de enero al 1 de febrero de 1939 (Mammach 1974).

Por tanto, alimentadas principalmente por las directrices marcadas por la ideología comunista, se encuentran en los textos de los artículos publicados en $F D$ reflexiones en torno a algunas ideas:

En primer lugar las reflexiones acerca de las circunstancias del escritor en el exilio: el escritor reconoce la situación internacional como algo excepcional y esto se hace tanto más patente cuanto el artista se encuentra en el exilio, fuera de su entorno de origen. Lion Feuchtwanger en el artículo «Problemas del trabajo del escritor 
en el exilio» recuerda a este respecto una clase a la que asistió en la universidad durante su juventud; en ella el profesor aseguraba que los escritores exiliados a lo largo de la historia no se veían influidos por el lugar en el que se encontraban al escribir y que lo más importante era la interioridad del artista, que le venía dada de nacimiento. Ya en aquel momento le extrañó esa teoría a Feuchtwanger y ahora, desde su propio exilio, muestra su desacuerdo con lo que oyó en aquella clase afirmando que el exilio no es una circunstancia secundaria para el escritor, sino que es la fuente de la que emanan sus obras, fuente que transformará no solo el contenido de sus escritos y la elección de temas, sino también su esencia.

El escritor se enfrenta a una serie de impedimentos y obstáculos extraordinarios como son la pérdida de su círculo de lectores $\mathrm{y}$, por tanto, la pérdida de su existencia económica:

Muchos escritores con un buen mercado en sus países no se venden en el extranjero a pesar de sus grandes dotes, bien porque su valor reside principalmente en lo lingüístico y este aspecto lingüístico no se puede trasladar, bien porque sus contenidos no interesan al lector extranjero [...] Es asombroso cuántos autores cuyos resultados han sido reconocidos por todo el mundo, se encuentran completamente sin medios y desvalidos a pesar de los esfuerzos más serios. (Feuchtwanger 1944: 27)

Por otro lado, el escritor se encuentra separado de su lengua materna, que cambia continuamente como organismo vivo: «En los diez u once años de nuestro exilio la vida ha continuado con mucha rapidez y ha necesitado para miles de nuevos conceptos miles de nuevas palabras. Nosotros oímos las nuevas palabras para estos nuevos fenómenos en la lengua extranjera» (Feuchtwanger 1944: 27).

Algunos escritores trataron de escribir en lengua extranjera, pero afirma Feuchtwanger que «nadie lo ha conseguido de verdad» y es una experiencia dolorosa para los escritores ver cómo una obra que ha sido concebida en la lengua materna no sale al mercado sino traducida porque «incluso la mejor traducción es extraña» (Feuchtwanger 1944: 27).

A la pérdida de los lectores y de la lengua se suma también la transformación a la que el escritor es sometido por el simple hecho de encontrarse en otro lugar, abocado a la aceptación de una realidad adversa, puesto que «encerrarse en el pasado muerto, apartarse de la vida real que le rodea a uno [...] merma la fuerza del poeta, la seca y la agosta» (Feuchtwanger 1944: 27). Por eso, la decisión más sabia para el citado autor sería la de adaptarse al presente.

Sin embargo, los escritores piensan que todas estas circunstancias extraordinarias y extremas que generan grandes tribulaciones traen también consigo, en ocasiones, algo positivo, ya que el escritor se encuentra en un mundo nuevo que no habría conocido si no fuera por el exilio. Estas novedades conllevan un gran enriquecimiento del mundo interior del artista que se verá reflejado en sus obras. Afirma también Feuchtwanger que «el sufrimiento hace más débiles a los débiles, pero a los fuertes los hace más fuertes» (Feuchtwanger 1944: 28), por eso el exilio es una «dura escuela». Trajo circunstancias muy desfavorables que llevaron a muchos artistas a la desesperación y al suicidio, pero a los que resistieron les enseñó y enriqueció. 
Abundan también en los artículos de $F D$ las reflexiones sobre el papel del arte y del escritor en la guerra. A aquellos que tuvieran la creencia generalizada de que las guerras se libran únicamente en los campos de batallas, les decía Bodo Uhse:

Quien piense que las guerras -y en especial esta guerra- sólo puede ser ganada con tanques, aviones y barcos acorazados, se equivoca. Se equivoca como aquel oficial que, con arrogante orgullo profesional, daba palmaditas en el hombro a un escritor: «Siga escribiendo con tranquilidad sus historias que entretanto haremos nosotros un poco de Historia». (Uhse 1943: 27)

Los intelectuales alemanes que colaboraban con la revista Alemania Libre, conscientes de su importancia, estaban convencidos de que la guerra se ganaba también con la literatura y con las palabras. Mientras unos luchaban con armas de fuego, otros lo hacían con el arma de las palabras. Por ello les parecía muy importante que el escritor se posicionara y cumpliera con su deber y obligación de participar en la guerra desde su profesión.

Como indica Uhse, la labor y el deber del escritor consistía, por una parte, en realizar un trabajo de aclaración y explicación de lo que estaba ocurriendo, debía ayudar a los lectores a entender el estado real de la situación bélica y a que, por ejemplo, la propaganda fascista, no consiguiera su objetivo de engañar al pueblo:

Nunca fueron tan necesarios los libros como ahora [...] si no se escriben esos libros que nos muestran claramente las alternativas ante las que nos encontramos, que nos capaciten a elegir sagazmente entre ellas, entonces se perderá la guerra, se perderá para toda la humanidad. (Uhse 1943: 27)

Por este motivo los escritores en la guerra no se limitan solo a influir a través de sus creaciones literarias, sino que toman parte activa para que la población alemana se levante y se rebele contra Hitler. De esta manera actúan y «penetran más profundamente en la vida de su pueblo» (Uhse 1943: 27). El escritor pasa a ser la conciencia del pueblo y tiene un papel imprescindible, como indica Ilja Ehrenburg: «Al escritor se le dio mucho, pero también se le exige mucho. No solo es responsable de cada una de sus palabras, sino que también se le responsabiliza de sus silencios» (Ehrenburg 1944: 27).

Pero, ¿cómo llevar a cabo esta tarea, esta responsabilidad? También hay respuestas a esta pregunta en los artículos de $F D$. El escritor no debería simplemente describir acontecimientos, sino prescribir y ayudar al pueblo a encontrar su esencia, a penetrar en el fondo de los acontecimientos y a reconocer al enemigo. Por eso es muy corriente que los escritores en la época de la guerra, y en el exilio, colaboren con los periódicos y sean ellos quien redacten artículos, puesto que

[...] en tiempos de paz el periódico es un medio de información y en la guerra es el aire que respiramos [...] el periódico es una carta que va dirigida personalmente a ti [...] de lo que aparece en el periódico depende tu destino personal. [Los escritores] se han ganado el derecho de hablar al pueblo. (Ehrenburg 1944: 27) 
El balance de lo que la guerra y el exilio estaba trayendo y había traído al arte y la literatura se va haciendo cuando se intuye cercano el final de la contienda. El mal se había extendido y había deshecho la identidad cultural alemana; internacionalmente el humanismo alemán había quedado cuestionado, por ello surgieron asociaciones de intelectuales como fue el propio movimiento $F D$, para crear un frente cultural que renovara todo hacia la construcción de una Alemania democrática.

Los escritores eran conscientes de que cuando acabara la guerra había que conseguir crear una nueva literatura, la de la «Nueva Alemania», y tenían muy claro que en todos los ámbitos de la literatura sería necesaria una profunda revisión:

Tanto la literatura especializada como la literatura escolar, la historiografía y la literatura política, pero también una gran parte de la literatura clásica y de la literatura de entretenimiento debe revisarse y editarse de nuevo. A todo esto han de sumarse también las obras producidas en el extranjero que han sido vetadas a los alemanes bajo el régimen de Hitler y que deberán ser publicadas en traducciones. (Janka 1945: 29)

El camino que escogieron para renovar la literatura fue evitando, en palabras de F. C. Weiskopf, «la así llamada huida de la realidad» (Weiskpf 1943: 27):

Si en el desarrollo de una nueva literatura alemana y un nuevo arte alemán no logramos encaminar las fuerzas liberadas hacia caminos de construcción y de realismo, es decir, llevarlas a estar en consonancia con las tareas sociales generales ante las cuales se encuentran las fuerzas antifascistas y democráticas del pueblo alemán, entonces la literatura estará condenada al fracaso [...] la demanda de realismo en la nueva literatura y el nuevo arte alemán es más que una simple exigencia estética. Si reconocemos la necesidad de poner una nueva literatura alemana al servicio de la educación del hombre alemán hacia la democracia y el humanismo, la exigencia del realismo se hace evidente por sí misma. Cada intento del artista por alejarse del realismo significa su salida de la realidad social. (Zimmering 1945: 27)

Fue, por tanto, el realismo socialista el camino en el que los escritores de la revista $F D$ creían para conseguir su finalidad de inmersión en la sociedad y, desde dentro, transformarla y dirigirla, en primer lugar, a la lucha y al combate contra el fascismo y el imperialismo y, tras la guerra, a la construcción de la Nueva Alemania. Toda desviación del realismo se veía como una pérdida de fuerza artística, como despilfarro y falta de compromiso social.

Según los textos publicados en $F D$, los escritores en lengua alemana durante la guerra y el exilio fueron conscientes de las circunstancias excepcionales que vivían y de cómo ellas y las dificultades que encontraron influían en su producción artística. El escritor comunista instrumentalizaba la literatura orientándola a un fin propagandístico que combatiera el fascismo e influyera en la población. Para ello tomó dos posturas: por un lado adoptó las formas del realismo socialista, por otro lado colaboró con la prensa, quedando muchas veces al margen del ámbito estrictamente literario. A la vez, es consciente del gran valor de lo producido durante la guerra y el exilio y reclama, una vez finalizada la contienda, que se haga una revisión y recuperación de todo lo que se ha prohibido en Alemania. 


\section{Referencias bibliográfícas}

BECHER, Johannes Robert (1944): «Eine Betrachtung über Kunst im Krieg». Freies Deutschland 3/10:27-28.

CAstro Leal, Antonio (1941): «Por una Alemania Libre». Freies Deutschland 1:2.

EHRENBURG, Ilja (1944): «Pflichten des Schriftstellers». Freies Deutschland 3/6:27-29.

FEUCHTWANGER, Lion (1944): «Arbeitsprobleme des Schriftstellers im Exil». Freies Deutschland 3/4:27-28.

JANKA, Walter (1945): «Buch und Verlag im kommenden Deutschland». Freies Deutschland 4/4:25-26.

MAMMACH, Klaus (1974): Die Berner Konferenz der KPD. Berlin: Marxistische Blätter.

MAYER, Paul (1946): «Leistungen des Verlages El Libro Libre». Freies Deutschland 5/5:25.

POHLE, Fritz (1986): Das mexikanische Exil. Ein Beitrag zur Geschichte der politisch-kulturellen Emigration aus Deutschland (1937-1946). Stuttgart: Metzler.

RiVERA OCHOA, María Clotilde (1987): Estudio de la revista Freies Deutschland. Órgano de difusión del movimiento Alemania Libre en México. 1941-1946. México D. F.: Instituto de Investigaciones Interculturales.

StEPHAN, Alexander (1979): Die deutsche Exilliteratur 1933-1945. München: Beck.

UHSE, Bodo (1943): «Der Schriftsteller und der Krieg». Freies Deutschland 2/3:27-28.

- (1943): «Verantwortung und Pflicht». Freies Deutschland 2/11:27-28.

- (1944): «Von Gegenwart und Zukunft der deutschen Literatur». Freies Deutschland 3/8:30.

Walter, Hans-Albert (2003): Deutsche Exilliteratur 1933-1950. Band 1.1. Die Vorgeschichte des Exils und seine erste Phase. Stuttgart: Metzler.

WeISKOPF, W. C. (1943): «Das Grauen sieht sich um. Bemerkungen zur deutschen Kriegsliteratur». Freies Deutschland 2/9:27-28.

ZIMMERING, Max (1945): «Deutsche Nachkriegsliteratur». Freies Deutschland 4/10:27. 